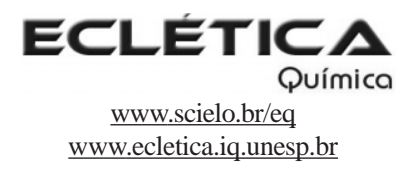

Volume 32, número 3, 2007

\title{
Constituintes químicos isolados dos caules de Michelia champaca L. (Magnoliaceae)
}

\author{
M. C. M. Monteiro ${ }^{1}$, I. H. Leptokarydis ${ }^{1}$, G. H. Silva ${ }^{1,2}$, V. C.da Silva ${ }^{1}$, V. S.Bolzani ${ }^{1}$, M. \\ C. M.Young ${ }^{3}$, M.N.Lopes ${ }^{1 *}$ \\ ${ }^{1}$ Departamento de Química Orgânica, Instituto de Química, Universidade Estadual Paulista “Júlio \\ de Mesquita Filho”, CP 355, CEP 14800-900, Araraquara, SP, Brasil. \\ ${ }^{2}$ Departamento de Química, Universidade Federal de Sergipe, CEP 49100-000, Aracajú,SE, Brasil. \\ ${ }^{3}$ Seção de Fisiologia e Bioquímica de Plantas, Instituto de Botânica, CP 4005, CEP 01061-970, \\ São Paulo, SP, Brasil. \\ *mnlopes@iq.unesp.br
}

\begin{abstract}
Resumo: O fracionamento cromatográfico da fase diclorometânica dos caules de Michelia champaca, forneceu quatro substâncias: álcool 4-O- $\beta$-D-glicopiranosídeo sinapílico, aldeído 4-O- $\beta$-D-glicopiranosídeo sinápico, siringaresinol e $N$-acetilnonaina. Extração ácido-base de uma nova porção do extrato bruto etanólico permitiu a identificação do alcalóide liriodenina. Os derivados fenilpropanoídicos e o alcalóide $N$-acetilnonaina estão sendo descritos pela primeira vez no gênero Michelia. O alcalóide liriodenina e a lignana siringaresinol apresentaram atividade antifúngica moderada.
\end{abstract}

Palavras-chave: Magnoliaceae; Michelia champaca; alcalóides; atividade antifúngica.

\section{Introdução}

Magnoliaceae compreende cerca de 10 gêneros e 220 espécies, sendo que a maioria ocorre no Hemisfério Norte, mas também são encontrados na Ásia (Índia e Malásia) e América do Sul (Brasil). No Brasil são cultivadas várias espécies dos gêneros Magnolia e Michelia, popularmente conhecidas como magnólia amarela [1].

A espécie Michelia champaca aparece como uma planta importante na medicina indígena [2]. É usada pela população para regular a menstruação, combater infecções da garganta e febres, artrites, reumatismos [3], como anticoncepcional [2] e como proteção pósparto [4]. Estudo farmacológico com o extrato da casca da raiz indicou propriedade inseticida potente contra a larva do mosquito Aedes egyptii [5].
Estudos fitoquímico e biológico com raízes [6], cascas da raiz [7] e folhas [6] de M. champaca levaram ao isolamento de lactonas sesquiterpênicas com significativa atividade antitumoral e permitiram estabelecer o perfil químico deste gênero como essencialmente terpênico [5], com predominância das lactonas sesquiterpênicas. KHAN e colaboradores [4] relatam, com base em triagem fitoquímica, a presença de alcalóides, flavonóides, taninos, saponinas, além de triterpenóides em folhas, sementes, caules e raízes de $M$. champaca. O flavonóide quercetina foi isolado das flores, pela primeira vez no gênero por KAPOOR e JAGGI [8].

Além da atividade antitumoral descrita anteriormente, alguns autores relatam também atividade antifúngica para a espécie. O extrato metanólico das flores 
de $M$. champaca mostrou forte atividade frente ao patógeno Staphylococcus aureus [9]. Os extratos diclorometânico e metanólico das folhas apresentaram atividade moderada frente a vários fungos patogênicos e fitopatogênicos [10]. Ainda, sementes, caules e raízes foram bioativos quando ensaiados com fungos patogênicos humanos [4].

Neste trabalho, descrevemos o fracionamento biomonitorado dos caules de $M$. champaca, buscando isolar e identificar os possíveis metabólitos secundários responsáveis pela atividade antifúngica detectada na fase diclorometânica quando bioensaiadas com os fungos fitopatogênicos Cladosporium cladosporioides e C. sphaerospermum.

\section{Materiais e Métodos}

Procedimentos experimentais gerais

Os espectros de RMN foram registrados em espectrômetro Varian AS 500 (500 MHz para ${ }^{1} \mathrm{H}$ e $125 \mathrm{MHz}$ para ${ }^{13} \mathrm{C}$ ). Foram utilizados os solventes deuterados $\mathrm{CDCl}_{3}$, DMSO- $d_{6}$ e $\mathrm{CD}_{3} \mathrm{OD}$, produzidos pela Acros, Aldrich ou CILL, sendo TMS o padrão interno. As colunas cromatográficas tiveram como fase estacionária sílica gel 60 H (70 - 230 mesh, Merck) e sílica C18 (43 $-60 \mu \mathrm{m})$. As análises em cromatografia em camada delgada foram realizadas em sílica gel $60 \mathrm{~F}_{254}$ (placa) e as manchas visualizadas por absorção da radiação UV, anisaldeído $2 \%$ em $\mathrm{H}_{2} \mathrm{SO}_{4}$ seguida de aquecimento, câmara de iodo e solução dragendorff. Os cromatogramas foram obtidos por Cromatografia Líquida de Alta Eficiência (CLAE), num aparelho Varian ProStar, com detector UV-Vis, registrados em microcomputador de $500 \mathrm{MHz}$ com processador Pentium II. Foram utilizadas colunas analíticas Phenomenex Luna C18 (5 ?m, 250 x 4,6 mm) e coluna preparativa Phenomenex Luna C18 $(10 \mu \mathrm{m}, 250$ x 21,20 $\mathrm{mm})$. Os solventes orgânicos utilizados para obtenção de extratos e fracionamentos foram das marcas Mallinckrodt, Synth e J. T. Baker (grau CLAE). Aágua (grau CLAE) foi obtida usando um sistema de purificação Milli-Q
Plus (Millipore). As soluções foram concentradas em evaporador rotativo Buchi R-114, sob pressão reduzida. Os espectros de massas de baixa resolução foram obtidos em um espectrômetro Platfform, no modo electrospray. Os espectros de infravermelho foram obtidos em um espectrômetro Nicolet-730 FT-IR. Material vegetal

Michelia champaca L. (Magnoliaceae) foi coletada no Campus da Universidade de São Paulo (USP), em Ribeirão Preto, São Paulo, Brasil e identificada pela Dra. Inês Cordeiro do Instituto de Botânica da Secretaria do Meio Ambiente do Estado de São Paulo.

Extração e isolamento dos constituintes químicos

Os caules $M$. champaca foram secos à temperatura ambiente, moídos e submetidos à percolação em etanol, resultando no extrato bruto (55,30 g), após evaporação do solvente sob pressão reduzida. $\mathrm{O}$ extrato bruto foi solubilizado em $\mathrm{MeOH} / \mathrm{H}_{2} \mathrm{O}(70: 30$, v/v) e submetico à partição com solventes de diferentes polaridades, fornecendo as fases hexânica (9,00 g), diclorometânica (13,04 g), acetato de etila (4,82 g), $n$-butanólica (11,90 g) e hidroalcóolica $(9,08 \mathrm{~g})$. Todas essas fases foram bioensaiadas para averiguação da atividade antifúngica.

Parte da fase diclorometânica (6,5 g), a qual foi a única a apresentar atividade, foi submetida à cromatografia em coluna (CC) usando sílica gel (70 - 230 mesh) e eluída com hexano/AcOEt e AcOEt/MeOH em misturas de polaridade crescente, fornecendo 22 frações, após análise por cromatografia em camada delgada. A fração 8 (690 mg, eluída em AcOEt/MeOH 80:20, v/v) foi submetida à CC utilizando sílica de fase reversa C18, eluída com $\mathrm{MeOH} / \mathrm{H}_{2} \mathrm{O}$ em gradiente decrescente de polarídade, obtendo-se 6 frações. A subfração 8.3 (74,2 mg, eluída em AcOEt/MeOH 40:60, v/v) foi submetida a CLAE preparativa em fase reversa C18 $\left(\lambda=254 \mathrm{~nm}, \mathrm{MeOH} / \mathrm{H}_{2} \mathrm{O}\right.$

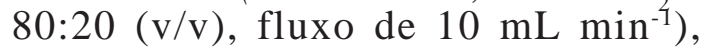


fornecendo as substâncias 1 (4,6 mg) e 2(3,7 mg). A fração 7 (420 mg, Hex/AcOEt 10:90, $\mathrm{v} / \mathrm{v}$ ) foi cromatografada em coluna de fase reversa $\mathrm{C} 18$, eluída em $\mathrm{MeOH} / \mathrm{H}_{2} \mathrm{O}$ em gradiente crescente de polaridade, obtendose 10 frações. A subfração $7.5(69,6 \mathrm{mg}$, $\mathrm{MeOH} / \mathrm{H}_{2} \mathrm{O} 65: 35$, v/v) foi submetida a CLAE preparativa $\lambda=247 \mathrm{~nm}, \mathrm{MeOH} /$ $\mathrm{H}_{2} \mathrm{O} 50: 50(\mathrm{v} / \mathrm{v})$, fluxo de $\left.17 \mathrm{~mL} \mathrm{~min}^{-1}\right) \mathrm{e}$ forneceu a substância 3 (9,3 mg). A fração 5 (380 mg, Hex/AcOEt 40:60, v/v) foi também submetida a cromatografia em fase reversa, à vácuo, fornecendo 11 frações. A subfração 5.8 (70,9 mg, $\mathrm{MeOH} / \mathrm{H}_{2} \mathrm{O}$ 80:20, v/v) foi submetida a cromatografia em camada delgada preparativa de fase normal, sendo eluída em AcOEt/MeOH 20:80 (v/v) e fornecendo a substância 4 (8,0 mg), após recristalização em $\mathrm{MeOH}$.

Uma nova porção do extrato bruto (25,0 g) foi solubilizada em $\mathrm{HCl} 5 \%$, após precipitação do material graxo com metanol. Fez-se, em seguida, extração com AcOEt, basificação da fase aquosa e novamente extração com AcOEt [11]. A fração alcaloídica $(247,4 \mathrm{mg})$ foi submetida à cromatografia em coluna utilizando $\mathrm{C} 18$, eluída em $\mathrm{MeOH} / \mathrm{H}_{2} \mathrm{O}$ em gradiente decrescente de polaridade, fornecendo a substância 5 (14,5 mg, MeOH/ $\mathrm{H}_{2} \mathrm{O}$ 70:30, $\mathrm{v} / \mathrm{v})$.

Bioensaio antifúngico

Estes ensaios foram realizados no laboratório do Instituto de Botânica de São Paulo sob a supervisão da Dra. Maria Cláudia Marx Young.

Suspensões contendo esporos dos fungos das espécies Cladosporium cladosporoides e Cladosporium sphaerospermum foram utilizadas como reveladores em cromatografia em camada delgada para a realização da bioautografia [12]. A amostra de extratos foi aplicada em cromatoplacas e eluída com solventes adequados. Após a evaporação dos solventes e localização das manchas por meio de absorção no ultravioleta, uma solução de glicose e sais contendo esporos do fungo foi nebulizada sobre as placas que foram incubadas à temperatura de $25^{\circ} \mathrm{C} \mathrm{em}$ câmara úmida e escura durante 2 a 3 dias. Após o tempo de incubação, observou-se o crescimento do fungo sobre a placa. Nos locais onde existem substâncias fungitóxicas surge um halo de inibição. Foi utilizado nistatina $(1 \mu \mathrm{g})$ como padrão positivo.

\section{Resultados e Discussão}

As fases hexânica, diclorometânica, acetato de etila, $n$-butanólica e hidroalcóolica, provenientes da partição líquido-líquido do extrato etanólico, foram bioensaiadas com os fungos Cladosporium cladosporoides e C. sphaerospermum, sendo que apenas a fase diclorometânica apresentou atividade moderada. Esta fase foi, então, fracionada em coluna cromatográfica de fase normal fornecendo dois derivados fenilpropanoídicos, álcool 4-O- $\beta$-D-glicopiranosídeo sinapílico (1) e aldeído 4-O- $\beta$-D-glicopiranosídeo sinápico (2), uma lignana siringaresinol (3) e um alcalóide $N$-acetilnonaina (4) (Figura 1).

Avaliação prévia do extrato bruto com revelador draggendorff, detectou a ocorrência de alcalóides na matriz vegetal. Assim, o extrato bruto foi submetido a extração ácido-base para obtenção de uma fração rica em alcalóides, que foi fracionada em coluna cromatográfica de fase reversa e levou ao isolamento de liriodenina (5) (Figura 1).

As estruturas de todas as substâncias isoladas foram determinadas através de métodos espectroscópicos (EM, IV e RMN de ${ }^{1} \mathrm{H}$ e ${ }^{13} \mathrm{C}$, uni e bidimensionais) e confirmadas através da comparação com dados da literatura.

Todas as substâncias isoladas do extrato ativo e a substância 5 foram submetidas a bioautografia com os fungos Cladosporium cladosporoides e $C$. sphaerospermum, e as substâncias 3 e 5 

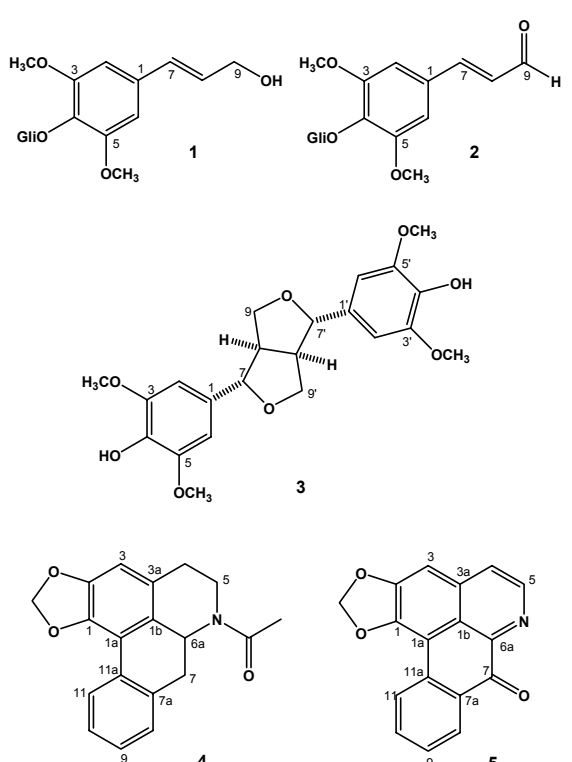

Figura 1. Substâncias isoladas dos caules de M. champaca.

apresentaram atividade na concentração de $100 \mu \mathrm{g}$ por ponto de aplicação. Álcool 4-O- $\beta$-D-glicopiranosídeo sinapílico (1). IV $v_{\max }(\mathrm{KBr}) \mathrm{cm}^{-1}: 3422$, 2923, 2846, 1645, 1587, 1453 e 1245; MS: $\mathrm{m} / z 395[\mathrm{M}+\mathrm{Na}]^{+}$; RMN de ${ }^{1} \mathrm{H}$ (DMSO$\left.d_{6}\right): \delta 6,72(2 \mathrm{H}, s, \mathrm{H}-2 \mathrm{e} \mathrm{H}-6), \delta 6,46(1 \mathrm{H}, d l$, $J=16 \mathrm{~Hz}, \mathrm{H}-7), \delta 6,33(1 \mathrm{H}, d t, J=5$ e 16 $\mathrm{Hz}, \mathrm{H}-8), \delta 4,90\left(1 \mathrm{H}, d, J=5 \mathrm{~Hz}, \mathrm{H}-1^{\prime}\right), \delta$ $4,85(1 \mathrm{H}, t l, J=5 \mathrm{~Hz}, \mathrm{H}-9), \delta 4,27(1 \mathrm{H}, t, J$ $=5,5 \mathrm{~Hz}, \mathrm{H}-4$ ' $), \delta 4,10(1 \mathrm{H}, t l, J=5 \mathrm{~Hz}, \mathrm{H}-$ $9), \delta 3,76\left(6 \mathrm{H}, s, \mathrm{OCH}_{3}\right), \delta 3,40(1 \mathrm{H}, m, \mathrm{H}-$ 6'), $\delta 3,20(1 \mathrm{H}, m, \mathrm{H}-3$ '), $\delta 3,13(1 \mathrm{H}, m, \mathrm{H}-$ $\left.2^{\prime}\right), \delta 3,00\left(1 \mathrm{H}, m, \mathrm{H}-5^{\prime}\right)$; RMN de ${ }^{13} \mathrm{C}$ (DMSO- $\left.d_{6}\right): \delta 152,7$ (C-3 e C-5), $\delta 133,8$ (C-4), $\delta 132,6$ (C-1), $\delta$ 130,2 (C-8), $\delta$ 128,4 (C-7), $\delta$ 104,5 (C-2 e C-6), $\delta$ 102,6 (C-1'), $\delta$ 77,2 (C-3'), $\delta$ 76,5 (C-5'), $\delta$ 74,2 (C-2'), $\delta$ 69,9 (C-4'), $\delta$ 61,4 (C-9), $\delta$ 60,9 (C-6'), $\delta$ $56,3\left(\mathrm{OCH}_{3}\right)$.

Aldeído 4-O- $\beta$-D-glicopiranosídeo sinápico (2). IV $v_{\max }(\mathrm{KBr}) \mathrm{cm}^{-1}: 2920,2850$, 1666 e 1400-1600; MS: $m / z 393[\mathrm{M}+$ $\mathrm{Na}^{+}$; RMN de ${ }^{1} \mathrm{H}$ (DMSO- $\left.d_{6}\right): \delta 9,64(1 \mathrm{H}$, $d, J=8 \mathrm{~Hz}, \mathrm{H}-9), \delta 7,62(1 \mathrm{H}, d, J=16 \mathrm{~Hz}$, $\mathrm{H}-7), \delta 7,09$ (2H, $s, \mathrm{H}-2$ e H-6), $\delta 6,89(1 \mathrm{H}$, $d d, J=8$ e $16 \mathrm{~Hz}, \mathrm{H}-8), \delta 5,08(1 \mathrm{H}, d, J=$ $7,5 \mathrm{~Hz}, \mathrm{H}-1$ ') $, \delta 3,81$ (6H, s, $\left.\mathrm{OCH}_{3}\right), \delta 3,38$
(1H, $m, \mathrm{H}-6$ '), $\delta 3,21$ (2H, $m, \mathrm{H}-2$ ' e H-5'), $\delta 3,13\left(1 \mathrm{H}, m, \mathrm{H}^{\prime} 4^{\prime}\right), \delta 3,06(1 \mathrm{H}, d d, J=2$ e 5,5 Hz, H-3'); RMN de ${ }^{13} \mathrm{C}$ (DMSO- $\left.d_{6}\right)$ : $\delta 194,2$ (C-9), $\delta$ 153,4 (C-7), $\delta$ 152,8 (C-3 e $\mathrm{C}-5), \delta 136,9$ (C-4), $\delta$ 129,4 (C-1), $\delta$ 127,9 (C-8), $\delta$ 107,1 (C-2 e C-6), $\delta$ 02,0 (C-1'), $\delta$ 77,4 (C-3'), $\delta$ 76,6 (C-5'), $\delta$ 74,1 (C-2'), $\delta$ 69,9 (C-4'), $\delta$ 60,8 (C-6'), $\delta 56,5\left(\mathrm{OCH}_{3}\right)$.

Siringaresinol (3). IV $v_{\max }(\mathrm{KBr}) \mathrm{cm}^{-1}$ : 3146, 2941, 2854, 1614, 1515 e 1458; MS: $m / z 441[\mathrm{M}+\mathrm{Na}]^{+}$; RMN de ${ }^{1} \mathrm{H}$ (DMSO$\left.d_{6}\right): .88,23(2 \mathrm{H}, s l, \mathrm{OH}$ e OH'), $\delta 6,59(4 \mathrm{H}, s$, H-2, H-2', H-6 e H-6'), $\delta 4,61$ ( $2 \mathrm{H}, d, J=4$ $\mathrm{Hz}, \mathrm{H}-7$ e H-7'), $\delta 4,15(2 \mathrm{H}, d d, J=5$ e 7 $\mathrm{Hz}, \mathrm{H}_{\mathrm{eq}}-9$ e $\left.\mathrm{H}_{\mathrm{eq}}{ }^{-9}\right), \delta 3,77(2 \mathrm{H}, d d, J=3,5 \mathrm{e}$ $7 \mathrm{~Hz}, \mathrm{H}_{\mathrm{ax}}-9$ e $\left.\mathrm{H}_{\mathrm{ax}}-9^{\prime}\right), \delta 3,75\left(12 \mathrm{H}, s, \mathrm{OCH}_{3} \mathrm{e}\right.$ $\left.\mathrm{OCH}_{3}{ }^{\prime}\right), \delta 3,04$ (2H, $m, \mathrm{H}-8$ e H-8'); RMN de ${ }^{13} \mathrm{C}$ (DMSO- $\left.d_{6}\right): \delta$ 147,9 (C-3, C-3', C-5 e C-5'), $\delta 134,7$ (C-1 e C-1'), $\delta 131,4$ (C-4 e C-4'), $\delta$ 103,6 (C-2, C-2', C-6 e C-6'), $\delta$ 85,3 (C-7 e C-7'), $\delta$ 71,0 (C-9 e C-9'), $\delta$ $56,0\left(\mathrm{OCH}_{3}\right.$ e $\left.\mathrm{OCH}_{3}{ }^{\prime}\right), \delta$ 53,6 (C-8 e C-8').

$N$-acetilnonaina (4): IV $v_{\text {máx }}(\mathrm{KBr}) \mathrm{cm}$ ${ }^{-1}$ : 2913 e 1626; MS: m/z $308[\mathrm{M}+\mathrm{H}]^{+}$; RMN de ${ }^{1} \mathrm{H}\left(\mathrm{CD}_{3} \mathrm{OD}\right): \delta 8,00(1 \mathrm{H}, d, J=$ $7,5 \mathrm{~Hz}, \mathrm{H}-11), \delta 7,20(1 \mathrm{H}, m, \mathrm{H}-8), \delta 7,13-$ $7,17(2 \mathrm{H}, m, \mathrm{H}-9$ ou H-10), $\delta$ 6,55 (1H, $s$, $\mathrm{H}-3), \delta 5,98\left(1 \mathrm{H}, d, J=1,5 \mathrm{~Hz}, \mathrm{O}-\mathrm{CH}_{2}-\mathrm{O}\right)$, $\delta 5,88\left(1 \mathrm{H}, d, J=1,5, \mathrm{O}_{-} \mathrm{CH}_{2}-\mathrm{O}\right), \delta 4,95$ $\left(1 \mathrm{H}, d d, J=3,5\right.$ e $\left.14,0 \mathrm{~Hz}, \mathrm{H}_{\mathrm{ax}}-6 \mathrm{a}\right), \delta 4,01$ $\left(1 \mathrm{H}, m, \mathrm{H}_{\mathrm{ax}}-5\right), \delta 3,18\left(1 \mathrm{H}, m, \mathrm{H}_{\mathrm{eq}}-5\right), \delta$ $2,97\left(1 \mathrm{H}, d d, \mathbf{J}=3,5\right.$ e $\left.14,0 \mathrm{~Hz}, \mathrm{H}_{\mathrm{eq}}-7\right), \delta$ $2,72\left(1 \mathrm{H}, m, \mathrm{H}_{\mathrm{ax}}-7\right), \delta 2,68(1 \mathrm{H}, m, \mathrm{H}-4), \delta$ $2,61(1 \mathrm{H}, m, \mathrm{H}-4), \delta 2,13(3 \mathrm{H}, s, \mathrm{O}=\mathrm{C}-$ $\left.\mathrm{CH}_{3}\right) ; \mathrm{RMN}$ de ${ }^{13} \mathrm{C}\left(\mathrm{CD}_{3} \mathrm{OD}\right): \delta 172,0$ $(\mathrm{C}=\mathrm{O}), \delta 148,8(\mathrm{C}-2), \delta$ 144,7 (C-1), $\delta$ 136,9 (C-7a), $\delta 132,6$ (C-3a), $\delta$ 129,6 (C$10), \delta 128,9$ (C-9), $\delta 128,8$ (C-11a), $\delta 128,3$ $(\mathrm{C}-8), \delta$ 128,0 (C-11), $\delta$ 126,6 (C-3b), $\delta$ 119,0 (C-1a), $\delta$ 108,5 (C-3), $\delta$ 102,3 (O$\left.\mathrm{CH}_{2}-\mathrm{O}\right) . \delta$ 52,2 (C-6a), $\delta$ 43,3 (C-5), $\delta$ 34,6 $(\mathrm{C}-7), \delta 31,8(\mathrm{C}-4), \delta 22,3\left(\mathrm{O}=\mathrm{C}-\mathrm{CH}_{3}\right)$.

Liriodenina (5): IV $v_{\text {máx }}(\mathrm{KBr}) \mathrm{cm}^{-1}$ : 2924, 2852, 1660 e 1400-1600; MS: m/z $276[\mathrm{M}+\mathrm{H}]^{+} ; \mathrm{RMN} d e{ }^{1} \mathrm{H}\left(\mathrm{CDCl}_{3}\right): \delta$ $8,86(1 \mathrm{H}, d, J=5,5 \mathrm{~Hz}, \mathrm{H}-5), \delta 8,60(1 \mathrm{H}, d$, $J=8,0 \mathrm{~Hz}, \mathrm{H}-8$ ou H-11), $\delta 8,56(1 \mathrm{H}, d d, J$ $=1,5$ e $8,0 \mathrm{~Hz}, \mathrm{H}-8$ ou H-11), $\delta$ 7,73 $(1 \mathrm{H}$, 
$d, J=5 \mathrm{~Hz}, \mathrm{H}-4), \delta 7,71(1 \mathrm{H}, m, \mathrm{H}-9$ ou H10), $\delta 7,55(1 \mathrm{H}, d t, J=1,5$ e $8,0 \mathrm{~Hz}, \mathrm{H}-9$ ou $\mathrm{H}-10), \delta 7,14(1 \mathrm{H}, s, \mathrm{H}-3), \delta 6,35$ (2H, s, O$\left.\mathrm{CH}_{2}-\mathrm{O}\right)$; $\mathrm{RMN}$ de ${ }^{13} \mathrm{C}\left(\mathrm{CDCl}_{3}\right): \delta 182,4(\mathrm{C}-$ 7), $\delta 151,7$ (C-1), $\delta 148,8(\mathrm{C}-2), \delta 145,2$ (C6a), $\delta$ 144,9 (C-5), $\delta$ 135,7 (C-3a), $\delta$ 133,9 (C-9), $\delta$ 132,9 (C-11a), $\delta$ 131,5 (C-7a), $\delta$ 128,8 (C-10), $\delta$ 128,6 (C-11), $\delta$ 127,3 (C8), $\delta$ 124,2 (C-4), $\delta 123,2$ (C-1b), $\delta$ 103,2 (C-3), $\delta$ 102,4 (O-CH $\left.\mathrm{CH}_{2}-\mathrm{O}\right)$.

\section{Conclusões}

O estudo químico dos caules de $M$. champaca levou ao isolamento e identificação de dois alcalóides, uma lignana e dois derivados fenilpropanoídicos, mostrando uma química diferenciada das raízes e sementes da planta, ricas em lactonas sesquiterpênicas. Este estudo contribuiu para o conhecimento dos metabólitos secundários de mais uma espécie da flora brasileira.
Os derivados fenilpropanoídicos e o alcalóide $N$-acetilnonaina estão sendo relatados pela primeira vez no gênero Michelia.Alignana siringaresinol jáhavia sido isolada da espécie Michelia fuscata, enquanto que liriodenina é comumente encontrada no gênero e em outras partes de M. champaca. A atividade antifúngica detectada na fase diclorometânica e confirmada na lignana 3 e no alcalóide 5 é um dado relevante, já que é a primeira vez que está sendo descrito para uma lignana. Substâncias com atividade frente aos fungos fitopatogênicos do gênero Cladosporium são de grande interesse para a agricultura.

\section{Agradecimentos}

Os autores agradecem à Fundação de Amparo a Pesquisa do Estado de São Paulo (FAPESP) pelo suporte financeiro e à bolsa de iniciação científica concedida a $\mathrm{M}$. C. M. M.

Recebido em : 11/05/2007 Aceito em : 08/06/2007

M. C. M. Monteiro, I. H. Leptokarydis, G. H. Silva, V. C. da Silva, V. da S. Bolzani, M. C. M. Young, M. N. Lopes. Chemical Constituents of Stems from Michelia champaca L. (Magnoliaceae)

Abstract: The fractionation of the dichlorometanic layer of dried stems of Michelia champaca afforded four substances: sinapyl 4-O- $\beta$-D-glucopyranoside alcohol, sinapyl 4-O- $\beta$-D-glucopyranoside aldehyde, syringaresinol and $N$-acetylnonaine. Part of the crude extract portion was submitted to acid-basic extraction allowed the identification of the alkaloid liriodenine. The two phenylpropanoids derivatives and the alkaloid $\mathrm{N}$-acetylnonaine were been reported for the first time from Michelia genus. The alkaloid liriodenine and the lignan syringaresinol showed moderate antifungal activity.

Keywords: Magnoliaceae, Michelia champaca, alkaloids, antifungal activity

\section{Referências}

[1] A. JOLY. São Paulo: Universidade de São Paulo (1975) 281.

[2] S. SHARMA, S. H. MEHTA. Indian Journal of Chemistry 37B (1998) 1219.

[3] M. P. CORRÊA. Rio de Janeiro: J. Di-Giorgio e Cia Ltda (1974) 31.

[4] M. R. KHAN, M. KIHARA, A. D. OMOLOSO. Fitoterapia 73 (2002) 744.

[5] U. JACOBSSON, V. KUMAR, S. SAMINATHAN. Phytochemistry 39 (4) (1995) 839.

[6] V. C. BALURGI, S. R. ROJATKAR, P. P. PUJAR, B. K. PATWARDKAN, B. A. NAGASAMPGI. Indian Drugs 34 (7) (1997) 415.

[7] V. K. SETHI, V. K. THAPPA, K. L. DHAR, C. K. ATAL. Planta Medica 50 (4) (1984) 364.
[8] S. KAPOOR, R. K. JAGGI. Indian Journal of Pharmaceutical Sciences 66 (4) (2004) 403.

[9] K. M. ELIZABETH, Y. A. S. J. LAKSHMI. Asian Journal of Chemistry 18 (1) (2006) 196.

[10] O. RANGASAMY, G. RAOELISON, F. E. RAKOTONIRIANA, K. CHEUK, S. URVERGRATSIMAMANGA, J. QUETIN-LECLEREQ, A. GURIB-FAKIM, A. H. SUBRATTY. Journal of Ethnopharmacology 109 (2007) 331.

[11] V. DA S. BOLZANI, A. A. L. GUNATILAKA, D. G. I. KINGSTON. Journal of Natural Products 58 (11) (1995) 1683.

[12] A. L. HOMANS, A. FUCHS. Journal of Chromatography 51 (1970) 327. 